\title{
Naming Virtual Identities: Patterns and Inspirations for Character Names in World of Warcraft
}

\author{
Christian Thurau ${ }^{1, *}$ and Anders Drachen ${ }^{2}$ \\ ${ }^{1}$ Visual and Social Media Group, Fraunhofer IAIS \\ christian. thurau@iais.fraunhofer.de \\ ${ }^{2}$ Department of Communication, Aalborg University \\ drachen@hum.aau.dk
}

\begin{abstract}
The abstract should summarize the contents of the paper and should People are increasingly interacting via online services - from forums, online communities, social networks and online computer games. While there has been considerable research on the motivations and communication of people online, as well as the social environment provided by online services, large-scale analyses of the virtual identities people use online are rare. In this paper, the first large-scale analysis of virtual identities in the massively multi-player online game WORLD OF WARCRAFT ${ }^{\circledR}$ is presented based on a dataset of nearly eight million avatar names and associated information. The results presented highlight the inventiveness of the names the users of WORLD OF WARCRAFT ${ }^{\circledR}$ express in terms of naming their virtual identities and the varied nature of the sources of inspiration for these names, which range from mythology, literature, popular culture, real-world names and others. The results also indicate that how players name their characters is influenced by the aesthetics and game function of the characters. The server type also appears to have an impact on player naming strategies, with role-playing servers forming a distinct cluster from Player-vs-Player type servers. Additionally, the analysis presented reveals that character name frequencies follow, similar to real-world names, a power law distribution.
\end{abstract}

Keywords: online identity, WORLD OF WARCRAFT®, character, avatar.

\section{$1 \quad$ Introduction}

A virtual identity or online identity is a social entity that an internet user establishes in an online community. One of the many forms of online communities in existence are formed by players interacting in the context of Massively Multiplayer Online Games (MMOGs) [1]. These digital games form entire virtual societies where users control avatars, or more precisely characters, interacting with the elements of the world as well as with other players $[2,3]$. Online identities are of scientific interest because technological development has enabled people to interact via networks on a worldwide basis, not the

\footnotetext{
* Corresponding Author.
} 
least through Internet based applications [4]. Digital online games form a vehicle of entertainment and socialization, and the number of users are increasing with the increased adoption of networked technologies in contemporary society [2, 5, 3]. Understanding how people operate online, the identities they assume, motivations for behavior and their interactions with each other and various environments (web forums, online games, social sites ...), is vital to the evaluate the effect network technologies have on human society as such. In the specific context of digital online games, the analysis of game characters (identities) is also of interest because it builds understanding of the people that play these games, whether for research- or commercial purposes. From the perspective of game development, analyzing characters informs design about the associations that the target audience have with specific names or features, thereby acting as a guide towards the design of e.g. non-player characters (artificial agents), where name, appearance and other features can be matched with the mindset of the players to evoke specific reactions. Hundreds of MMOGs are currently in existence, although one dominates the market in terms of the sheer number of people that play the game and form part of the associated online community: World of Warcraft (released 2003) with its reputed 12 million players ${ }^{1}$. WORLD OF WARCRAFT® (WOW) is fantasy-themed. The players control a customized character, which forms the main vehicle for interacting with the virtual environment, and the creation of which is a source of considerable attention from the players [6]. The game poses a variety of challenges, including various types of quests, teamwork assignments, as well as an in-game economic system [7]. The player will at any one time control one character, but can have multiple characters. Rather than interacting within the same virtual environment, players are distributed among hundreds of copies of the game (also called realms) [8].

Characters in MMOGs such as WOW have several traits that appear with more or less consistency across games of this type, notably: an appearance (typically a 3D avatar), specific abilities related to the game mechanics (which determine their effectiveness in different contexts), a name (usually the only truly unique feature of a character in a specific game), relationships with other characters, specific behaviors, and MMOGs typically contain some sort of character development system, although this can be limited to changes in e.g. appearance. Finally, characters often have a degree of persistence, which can cover years of real-time [2, 3]. In WOW, the characters the players control have names that are unique within the context of a specific realm. Creating a character is a process of making a set of foundational gameplay choices, e.g. a player must choose a race (e.g. Human, Orc), which impacts on the appearance of the character, and a class (e.g. Shaman, Mage), which impacts on the abilities of the character (e.g. a Warrior is a close-combat specialist). Previous research on WOW $\mathrm{R}$ indicates that character name, appearance and functionality are somehow linked (e.g. [9]), however, previous research is limited in terms of sample size and adopts informal methodologies. Here a mixed-methods explorative approach is adopted, based on 7.93 million characters from WOW. This is the first time - to the best knowledge of the authors - that a large-scale investigation of games has focused on virtual

${ }^{1}$ According to the developer, Blizzard Entertainment: http://eu.blizzard.com/en-gb/company/press/pressreleases.html?101007 
identities. The methodology presented thus forms a contribution in its own right, in addition to several new insights, e.g. that the appearance and game function influences the names chosen for characters. Essentially, the names players give to characters on Role-Playing, RP, realms show differences from those of Player-vs-Player, $\mathrm{PvP}$, and Player-vs-Environment, PvE, realms. The sources of inspiration for character names were investigated for a sample of 120,000 characters, indicating diverse sources of inspiration for character names, and that names with a negative semantic meaning (e.g. "Nightmare") are more than six times as common as those with a positive meaning (e.g. "Hope").

\section{Previous Work}

There is a substantial amount of research on virtual identities in online environments, notably Internet web communities, although most of this from sociological and communication perspectives, with limited large-scale quantitative work [11]. To the best knowledge of the authors, large-scale (millions of users) computational studies of the names people adopt in virtual environments, web communities and similar, have not been carried out before. However, Massively Multi-player Online Games (MMOGs) as WOW have for the past decade attracted the attention of researchers, notably sociologists, etnographers, design researchers as well as people working with communication and economy (e.g. [2, 12, 5, 6, 3]).

The investigation of the players of MMOGs have also included the mining large-scale user-telemetry datasets, mainly for the purpose of either investigating the players themselves [13, 14], informing game development [15] or for the development and testing of data mining algorithms for complex datasets [16]. Although prior large-scale work on user telemetry mining has not focused on the identity aspect or names specifically, it is worth mentioning as future research may show that e.g. motivations for play and the choices made during character generation correlate. User telemetry is in the context of digital games quantitative data about player-game and player-player interaction, and is generally compiled in databases from logs provided by game clients, or alternatively by extracting data directly from game servers $[15,14,16]$. Any action initiated by either a player or the game software can be recorded, from low-level data such as button presses to in-game conversations. In the current case, the user telemetry is mined from a repository (the WOW census site) which obtains the data from the game. The relationship between players and characters in digital games has formed one of the main lines of investigation [2, 17, 18]. However, while the virtual identities of players and the social dynamics of game world communities have been the subject of hundreds of research papers, only a limited number of these consider the names of player characters in detail: [19] examined the cross-modal compensation between name and visual aspect in WOW avatars (player characters), based on a nonrandom sample of 1261 names. The study was limited to consider whether name properties were affected by the visual aspect (human vs. non-human differentiation only) of the character. A main conclusion was that female names contained more 
vowels than male names. [20] investigated the relationship between the "capacity" of characters (their skills and abilities in terms of game mechanics) and their appearance. The study is qualitative and the sample size is unspecified, but the author concludes that capacity and appearance is connected and provides a means of player identification. [9] Collected a non-random sample of 1366 names of WOW characters, including race, class, gender and level information at the time of observation. The work appears to be purely observations, as the author does not write how the names were analyzed nor any statistical properties of the dataset, but conclude that names for all races in the game were "equally strange, odd and funny", and that there was no observable difference between low-level and high-level characters.

Table 1. Basic statistics of the character name dataset. We collected a total of $7,938,335$ character names. The realm-types specify if the particular world is hostile (PVP), friendly (PVE), or targeted towards role playing (RP) which prohibits non-medieval character names.

\begin{tabular}{lrrlrr}
\hline & (a) & \multicolumn{2}{c}{ Location } & & \multicolumn{2}{c}{ (b) } & Realm type \\
\hline Location & \# characters & \# unique names & Realm type & \# characters & \# unique names \\
All & $7,938,335$ & $3,803,819$ & PVP & $3,128,464$ & $1,869,481$ \\
Europe (EU) & $3,378,589$ & $1,820,269$ & PvE & $3,884,205$ & $2,207,478$ \\
United States (US) & $4,559,746$ & $2,495,960$ & RP & 619,892 & 499,481 \\
\hline
\end{tabular}

[9] Also describes a hypothesis that names between RP-servers and other server types would differ, but is not able to verify this (any methodology used is not described). The author does, however, describe a variety of sources or inspirations for names, including various mythologies, but no analysis of e.g. relative frequencies of these sources. [9] Also communicated with an undisclosed number of players about the sources of the inspirations for their character names, obtaining a variety of explanations. He highlights that: "in WOW $\mathrm{R}$ it is the name more than appearance that distinguishes avatars, and thus players, from each other", referring to the limited customization options available in terms of character appearance, but the virtually unlimited options in naming characters, despite the restrictions imposed by the game's Terms of Use. While an in-depth study of a few cases, the work of both [9] and [20] lacks evaluation and the sample size to hold substantial explanatory power.

\section{Statistical Analysis}

We gathered all 7,938,335 character names from the 50 largest guilds on each EU and US WOW realm from the WOW census site www.warcraftrealms.com. 4,559,746 character names belong to US players, 3,378,589 to EU players. The amount of character names distributes almost equally on Player-vs-Player (PVP) and Player-vsEnvironment (or Engine) (PVE) realms. Role-Playing (RP) realms make only less than $10 \%$ of the total number of names. Detailed numbers on the used dataset can be seen in Table 1). For a statistical analysis of the distribution of the name frequencies, we first preprocessed the dataset and replaced any special characters (e.g. the acute accent "”). Then, we computed histograms of name occurrences for each realm or realm-type. Overall, we computed 35 different histograms from selections of the 
original data. The selections correspond to realm types (All realms, US realms, EU realms, PVP realms, US-PVE realms, . . .); the various classes (Priests, Druids, . . .) and races (Trolls, Orcs, Humans, Undead, ... .). The total number of counted character names varies among the different categories, going from a few hundred thousands to approx. 8 million for the "All realms" category. The number of unique character names follows a logarithmic function dependent on the total number of characters, as can be seen in Fig. 3(b) i.e. that increasing the number of names in a sample provides diminishing returns in terms of increasing the number of unique names.

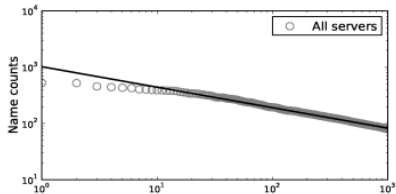

(a) All realms

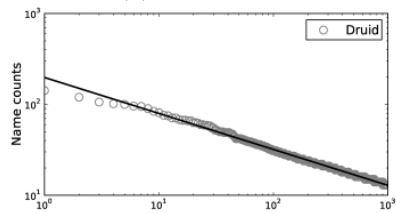

(d) Druid class

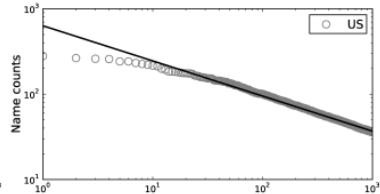

(b) US realms

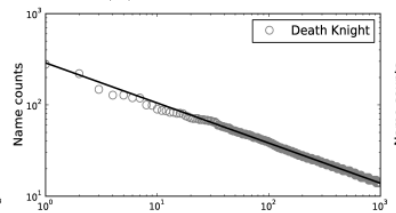

(e) Death knight class

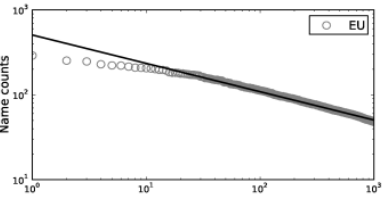

(c) EU realms

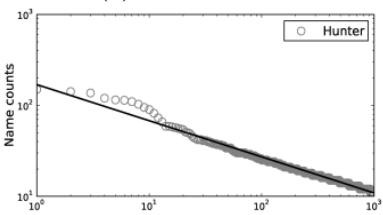

(f) Hunter class

Fig. 1. Rank-frequency plots for different selections of the character name dataset. Both axes are logarithmically scaled. The $y$-axis denotes the total count for a particular name, the $\mathrm{x}$-axis denotes the rank index (the left most ranked name has the highest number of occurrences).

\subsection{Character Name Frequencies}

It is known that the frequency of family names in many countries follows a power law distribution. Informally, this means that the second most popular name appears half as often as the most popular name, the third most popular names appears half as often as the second most popular name, and so on. Formally, a quantity $\mathrm{x}$ obeys a power law if it is drawn from a probability distribution $p(x) \alpha x^{-\alpha}$, where $\alpha$ is the scaling parameter or exponent [21]. Power laws are commonly found among various quantities in physical, biological, and social systems. In certain contexts they are referred to as Zipf's or Benford's law (see e.g. [22]). Popular examples include the number of citations of scientific papers, word frequencies in books, or populations of cities. As a thorough review of power law distributions is beyond the scope of this work, we recommend [23] for a general introduction and insights in their explanation. While it is reasonable to assume a power law distribution for the character name frequencies, after all this seems to be very similar to the distribution of real-world family names [10], there are two important preconditions that make character names differ from family names: First, a character name might only appear once per realm. While players are sometimes using special characters (e.g. German umlaut) to get around this limitation, it still influences the possible choices for a name. Second, unlike family names or real 
names, the choice of a name is unrestricted, only certain names that violate the Blizzard Naming Policy are forbidden (but see below).

For validating the power law distribution assumption, we fit a linear function to the logarithmically scaled rank-frequency plots of the 1000 most frequently occurring names. Some resulting rank-frequency plots of the name frequencies can be seen in Fig. 2, and of the fitted linear function in Fig. 1.

The power law assumption holds for all 35 separate data selections. The overall worst accuracy for the linear predictor is achieved for the "All realms" category and achieved a standard error $\sigma$ of 0.0025 , see Fig. 1(a) $\left(\sigma=\sqrt{\sum_{i}^{n}\left(x^{i}-x^{\prime} i\right.}\right) 2 / n$, where $x^{\prime}$ denotes the predicted value and $n$ the number of considered names). Note that $\sigma$ is computed for logarithmically scaled values (also note that fitting a linear function is not the most accurate way of verifying a power law assumption [21], however, given the very high accuracy of the linear fit this is negligible). A first important finding from the analysis is that all character name frequencies follow a power law distribution. With an increasing total number of character names, e.g. for the "All realms" category, it shows that the standard error $\sigma$ is increasing. However, the approximation accuracy is still high. A possible reason for the increased $\sigma$ might be the constraints imposed on character names (e.g. the one name per realm constraint).

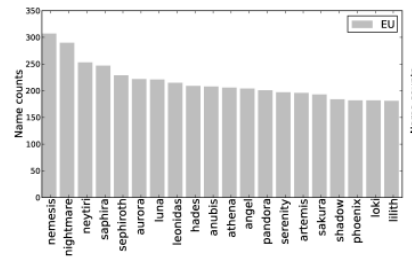

(a) EU realms

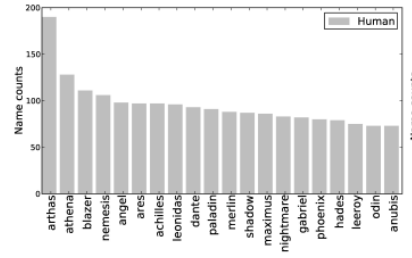

(d) Human race

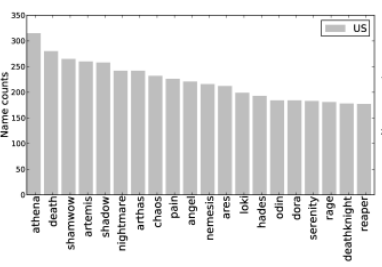

(b) US realms

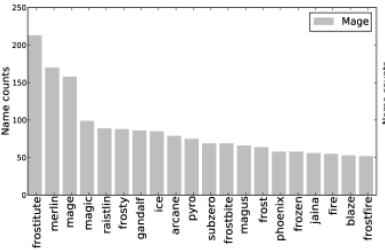

(e) Mage class

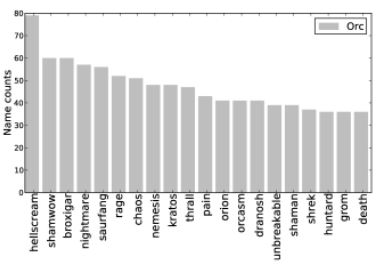

(c) Orc race

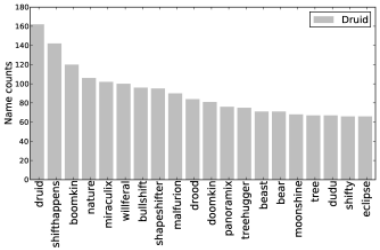

(f) Druid class

Fig. 2. Ranked character name counts for different selections of the character name dataset. Each plot shows on the $y$-axis the total number of name occurrences, and on the $\mathrm{x}$-axis the corresponding name. The $\mathrm{x}$-axis is sorted s.t. the most frequently occurring name comes first. It can be seen that the name counts roughly follow a power-law distribution.

\subsection{Character Name Distributions}

For comparing the name distributions across categories, we embedded the histogram L1-distances into a 2D space by means of Isomap [24]. The resulting embedding can be seen in Fig. 3(a). Categories (different realm-types or classes) that are close 
together also have a similar character name distribution. Categories that are far away from each other have a smaller overlap in character names. Fig. 3(a) shows two important findings: First, the names on US and EU realms have a rather large distances. However, this does not apply to Role-Playing (RP) realms. The plot shows that RP realms seem to have a larger overlap for US and EU realms. Second, there seems to be a separation in character names between the more human-like (Human, Blood Elf, Night Elf, Draenei) and less human-like ("bestial")/human-proportioned (Troll, Undead, Orc, Tauren, Dwarf, Gnome) races. A possible explanation for this might be the uneven distribution of male and female characters and names for certain races. We found that for the less humanlike races, there is a tendency towards male characters, whereas for the more human-like races we observed more female characters. However, in showed that this is not the reason as for only male and only female character names the results look almost identical.
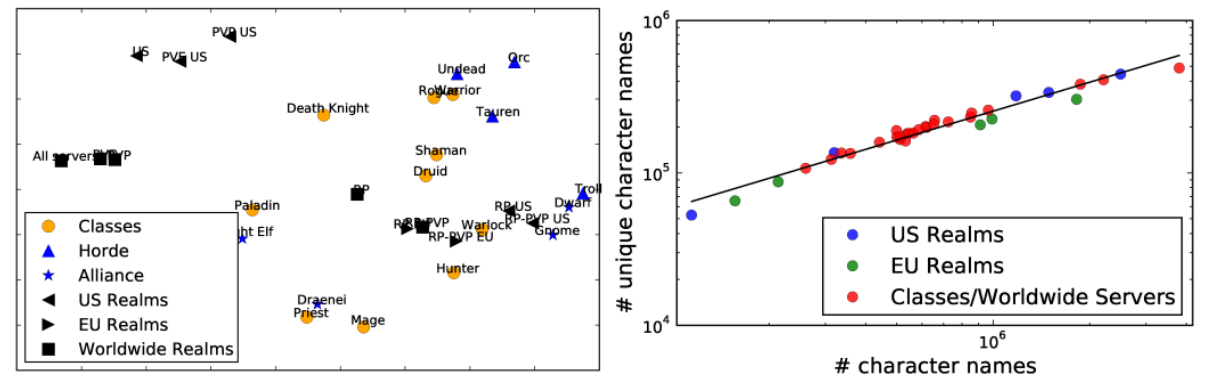

Fig. 3. Fig. 3(a) shows 2D-Isomap projection of histograms of character names for different categories. Categories which are closer together (e.g. "Undead" and "Orc" in the upper right part corner) have a larger overlap in character names. Fig. 3(b) shows the ratio of unique names and total number of names for different categories: the $\mathrm{x}$-axis shows the number of unique names per category, the $y$ shows the number of players. Both axes have a log-scaling.
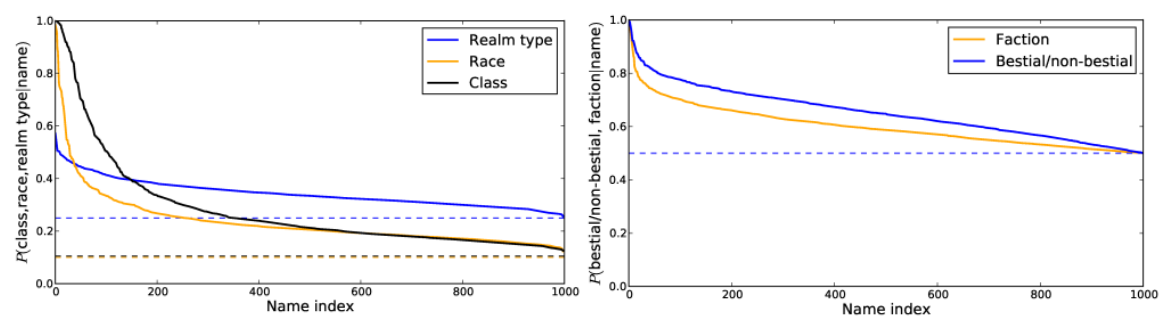

Fig. 4. Probabilities for a specific class, race, server type, faction (Horde or Alliance), and human-like/less human-like race given the character name. The probabilities are evaluated for the 1000 most popular names and the highest probability is selected. Afterwards, the names are sorted according to their highest probability. It can be seen that, for example, the player class (left figure) and faction (right figure) influence character names to a certain extent. The dashed lines denote probabilities for random selection for the corresponding feature. 
To further investigate the separation in character names according to races or classes, we evaluated a simple predictive model for character names. The idea is to estimate conditional probabilities of a particular class, race, or server type given a character name, e.g. how likely is it that a character named "Gimli" is a "dwarf"? We estimated these probabilities for the 1000 most popular names as we here have a sufficiently large sample set (probabilities are normalized by class/race/server-type priors s.t. we do not punish seldom selected classes etc.). In particular, we were interested in the uniqueness of a specific name with respect to a particular class or race. This indicates which variables influence player naming decision. Some results can be seen in Fig. 4 . The most influential features appear to be the player class, player race (which of course influences human-likeness), however, the server type and faction also give hints on player naming decisions.

\section{Sources of Inspiration}

In order to evaluate the sources of inspiration for the names people give their characters in WOW, a variety of computational approaches were considered. However, the sheer variety of character names in the dataset (approximately 3.9 million. Note that umlauts were removed during pre-processing, so any names like "Gändälf" and "Gandalf" were aggregated) eliminated all standard computational approaches. We therefore opted for an explorative categorical coding [25] approach for a sample of 1,000 character names. Rather than randomly selecting these, the most common names in the dataset were chosen. This has the effect of increasing the effective sample size, as the 1,000 most common names in the dataset comprise the names of 128,058 characters. While a respectable sample size (roughly 100 times larger than e.g. [9]), this represents only $1.6 \%$ of the total dataset, which means that making inferences from the sample to the entire dataset should only be done on an indicative basis. 38 coding categories were defined, 10 occurs only once, 11 from 2-10 times (Table 2). Determining the inspirational source for a character name is an exercise in estimation, due to the sheer variety of potential sources. For example, the name "Raziel" stems from cabalistic theory and is the name of an angel. However, the same name has also been used for computer game characters, in fiction books etc. A similar problem exists for the names associated with Greek, Roman, Germanic, Nordic, Mesopotamian and Japanese mythology located, e.g. "Odin", "Athena" or "Amaterasu". These names have been used in different contexts (film, literature, games) and in these cases it was necessary to code the names after the original myth they stem from. This does not mean that the players using these names were inspired by reading books about mythology, but at least it provides an accurate point of origin for the names. In comparison, names such as "Naruto" and "Luthien" are easier to place. The first stems from a major Japanese manga series of the same name, whereas the latter stems from J. R. R. Tolkien's fantasy world. Neither name has been used in other major contexts, which means that a best case for the source of inspiration of the names is the major popular culture/literature works they originally appear in. The most common category is real-world normal names (186), e.g. "Ella", "Yuki" and "Jack". As with 
names originating in mythology, at least a proportion of these normal names can also be found in popular culture (film, TV, games etc.), and it therefore cannot be determined what the exact source of inspiration for the players adopting these names were. In order to explore the potential sources of character names, searches were performed on Google and web-based resources like Wikipedia.org. Care was taken to explore multiple potential sources in depth where these were apparent. 697 names could be identified and classified in this manner Table 2. The remainder were nouns and verbs of unspecified nature but semantic meanings. Tentatively, these can be categorized into three classes: 1) Negative (156 counts): These are names that have "negative/dark" connotations, for example "Nightmare", "Sin", "Fear" and "Requiem"; 2) Positive (22 counts): These are names with distinct positive/'light" connotations, e.g. "Hope", "Love", "Pure"; 3) Neutral (125 counts): These are names with no obvious negative or positive connotations, e.g. "Who", "Moonlight", "Magic" and "Snow". Future analysis will go into more detail with the semantic meaning of these categories.

Table 2. Coding category and corresponding frequency counts based on the 1000 most popular character names

\begin{tabular}{ll}
\hline Count & \multicolumn{1}{c}{ Coding category } \\
\hline 186 Normal name & Common name \\
156 Negative (semantic) & Unspecified noun or verb with negative connotations \\
125 Neutral (semantic) & Unspecified noun or verb with neutral connotations \\
79 popular culture (film and TV) & $\begin{array}{l}\text { Reference to a character from a movie or television show } \\
\text { (including animated series) }\end{array}$ \\
& Name of an entity in Greek mythology \\
73 Greek myth & Reference to a character in a digital game \\
47 popular culture (games) & Reference to a character in a comic book series \\
43 popular culture (comics) & Reference to a character class in WOW \\
39 Class reference & Reference to fantasy literature \\
39 Fantasy literature & Unspecified noun or verb with positive connotations \\
22 Positive (semantic) & Name from the Bible \\
18 Biblical & Reference to the function of the character class \\
18 Game functional & Reference to a name/place in the WOW world or history \\
18 WOW myth & Typically the name of an angel in Jewish mysticism \\
17 Kabbalistic belief & Humoristic name \\
15 Humor & Name of an entity in Nordic mythology \\
15 Nordic mythos & Reference to the performance of the player's character \\
11 Performance reference & Name of an entity in Egyptian mythology \\
10 Egyptian myth &
\end{tabular}

In summary, a number of conclusions can be drawn: 1) Regular real-life names are the most common (186 counts). 2) Names with negative semantic meaning were more than six times as common as those with positive meaning. The reason for this tendency can only be speculated about without an in-depth inquiry among the players of WOW, but is possibly related to the emphasis on conflict in the game. 3) 164 names have an origin in mythology or the Bible, with Greek mythology being by far the most popular (73 counts). 4) 174 names in the list relate to popular culture, mainly characters from digital games (e.g. "Zelda", "Raiden", "Kerrigan"), films, television series (manga series like "Bleach" and "Naruto" being particularly popular), and comic book superheroes (e.g. "Goku", "Hawkeye", "Electra"). 5) Of the 39 references to 
fantasy literature, only four do not stem from the works of J. R. R. Tolkien. 6) Character names to a substantial degree are in breach of the Terms of Use of $\mathrm{WOW}^{2}$. The restrictions specify that names can be only one string of text, with no special characters and of limited length. This is enforced during character creation. Names may not be offensive, or belong to a popular culture figure from e.g. film or TV (something players blatantly ignore), contain or be similar to a trademark, have religious significance, be related to a real-world person (131 counts of "Chucknorris" occur in the dataset) or related to any of Blizzard's Warcraft products, but the name "Sylvanas" occurs 129 times and "Jaina" 148 times, both names of WOW characters) ${ }^{3}$. The restrictions on names - apart from the physical limits on text string length etc. - appear to be somewhat unenforced, maybe due to the challenge of collating and updating lists of all potential names in breach of the Terms of Use, and cross-comparing these with the millions of player character names, would be extremely resource demanding.

\section{Conclusion}

The study of online games and the people who play and interact through them is of importance to the general evaluation of how new technologies impact on human behavior $[2,5,3,1]$. Investigating the identities of players in online games is of direct value for two main reasons: 1) It informs about the people who play these games whether as objects of research inquiry or for target group analysis in the context of game marketing; 2) It provides information for the design and development of games in terms of how names, appearance and other features of characters are perceived by the players, and thus e.g. guidelines for how to utilize these features in the design of non-player characters (artificial agents) to evoke particular associations in the players. The research presented here provides evidence for a staggering variety in the names chosen by players of WOW R - 3.8 million unique names in a sample of 7.93 million, which is notably remarkable given the restrictions imposed. It highlights the imaginativeness employed in naming characters, indirectly supporting earlier work such as [6, 19] in concluding that the choices made during the character creation process are important to the players. Character names also follow the same kind of logdistribution as real-world names - despite MMOGs being only about 1.5 decades old and restrictive in terms of name choices. The analysis also shows that the appearance of a character and its functionality within the confines of the game plays a role when a player of WOW selects a character name - to a degree where it may be possible to develop predictive models which can assist game developers to evaluate player populations based on character names and similar sparse data.

The link between name and character features is also evident in the marked difference in the names given to characters from the "non-bestial" vs. "bestial" races (including, surprisingly, the otherwise non-bestial but also non-humanly proportioned Dwarves and Gnomes), where predictive modeling indicates a strong differential(Fig. 4); or the differences in names when viewed across character classes (Fig. 2). It is also

\footnotetext{
${ }^{2}$ http://us.blizzard.com/en-us/company/legal/wow_tou.html

3 The names can also be found in the WOWCensus system www.warcraftrealms.com
} 
shown how the kinds of rules that provided for interaction between players impact on how they name their characters - as is evident in the contrasts between PvP and RP servers, similar for choice of race. The work presented here also emphasizes the variety of inspirational sources evident in the sampled character names.

The work presented also posits new questions, e.g. why certain types of name inspirations are more common than others, and why there is variance in the diversity of names within specific classes of the game, e.g., characters of the Mage class exhibit a higher variety of names than for the other classes. There is also a variety in how many times the most popular names occur - for example, the name "Hellscream" is the most popular among the Orc characters (almost 80 counts), but for the other races the most popular name is more frequent (e.g. Draenai over 130 counts of "Neytiri" and for Humans over 180 counts of "Arthas"). The quantitative analysis presented here does not permit any conclusions to be made about these questions, which require communication with the players directly, but opens up the perspective that the distributions of names is not random but highly affected by the environment they are used in relation to. Possibly, this is also the case for other types of online environments, although the lack of large-scale analysis of online identities in e.g. web forums or social networks prevent any conclusion to be made in this regard.

Due to space limitations, we could only present a fraction of results developed based on the described dataset. Additional results based on another dataset from the WOW

armory will be reported on elsewhere or in an extended version of this paper (please note that results from other data sources support results presented here). In addition, future work will focus on adding to the details of the results presented, investigate approaches such as topical profiling (the definition of association strengths between words via mining the internet) for automating name classification rather than explorative coding, add additional character features to the analysis and examine correlations between sources of inspiration for names and character features, aiming to improve the predictive potential of name-based classification.

Acknowledgements. The authors would like to extend their warmest gratitude to Dr. Menno Van Zaanen and Dr. Thea Drachen for discussions, advice and feedback.

\section{References}

1. Bohannon, J.: Game-Miners Grapple With Massive Data. Science 330(6000), 30-31 (2010)

2. Steinkuehler, C., Williams, D.: Where Everybody Knows Your (Screen) Name: Online Games as Third Places. J. of Computer-Mediated Communication 11(4), 885-909 (2006)

3. Pearce, C., Artemesia, A.: Communities of Play: Emergent Cultures in Multiplayer Games and Virtual Worlds. The MIT Press, Cambridge (2009)

4. Madden, M., Smith, A., Vitak, J.: Digital footprints: online identity management and search in the age of transparency. Pew Research Center (2007)

5. Castranova, E.: Exodus to the VirtualWorld: How Online Fun Is Changing Reality. Palgrave Macmillan, Basingstoke (2007) 
6. Caplan, S., Williams, D., Yee, N.: Problematic internet use and psychosocial well-being among mmo players. Computers in Human Behavior 25(6), 1312-1319 (2009)

7. Bohannon, J.: Slaying monsters for science. Science 320(5883), 1592 (2008)

8. Corneliussen, H.G., Rettberg, J.W. (eds.): The MIT Press, Cambridge (2008)

9. Hagström, C.: Playing with names: Gaming and naming in world of warcraft. In: Vorderer, P., Bryant, J. (eds.) Digital Culture, Play, and Identity a World of Warcraft Reader, pp. 265-286. The MIT Press, Cambridge (2008)

10. Zanette, D.H., Manrubia, S.C.: Vertical transmission of culture and the distribution of family names. Physica A: Statistical Mechanics and its Applications 295(1-2), 1-8 (2001)

11. Wiszniewski, D., Coyne, R.: Mask and identity: The hermeneutics of self-construction in the information age. In: Renninger, K.A., Shumar, W. (eds.) Building Virtual Communities, pp. 191-214. Cambridge University Press, Cambridge (2002)

12. Bainbridge, W.S.: The scientific research potential of virtual worlds. Science 317(5837), 472-476 (2007)

13. Ducheneaut, N., Moore, R.J.: The Social Side of Gaming: A study of interaction patterns in a Massively Multiplayer Online Game. In: Proceedings of the 2004 ACM Conference on Computer Supported Cooperative Work (2004)

14. Williams, D., Consalvo, M., Caplan, S., Yee, N.: Looking for gender (lfg): Gender roles and behaviors among online gamers. J. of Communication 59, 700-725 (2009)

15. Drachen, A., Canossa, A., Yannakakis, G.N.: Player modeling using self-organization in tomb raider: Underworld. In: Proceedingas of IEEE Computational Intelligence in Games, pp. 1-8 (2009)

16. Thurau, C., Kersting, K., Bauckhage, C.: Convex non-negative matrix factorization in the wild. In: Proc. IEEE Int. Conf. on Data Mining (2009)

17. Tychsen, A., Hitchens, M., Brolund, T.: Character play: the use of game characters in multiplayer role-playing games across platforms. Computers in Entertainment 6(2) (2008)

18. Vorderer, P., Bryant, J.: Playing Video Games. Lawrence Erlbaum Associates, Mahwah (2006)

19. Guitteon, M.J.: Cross-modal compensation between name and visual aspect in socially active avatars. Computers in Human Behavior 26(6), 1772-1776 (2010)

20. Tronstad, R.: Character identification in world of warcraft: The relationship between capacity and appearance. In: Vorderer, P., Bryant, J. (eds.) Digital Culture, Play, and Identity a World of Warcraft Reader, pp. 249-264. The MIT Press, Cambridge (2008)

21. Clauset, A., Shalizi, C.R., Newman, M.E.J.: Power-law distributions in empirical data. SIAM Review 51(4), 661-703 (2009)

22. Pietronero, L., Tosatti, E., Tosatti, V., Vespignani, A.: Explaining the uneven distribution of numbers in nature: the laws of Benford and Zipf. Physica A 293(1-2), 297-304 (2001)

23. Newman, M.E.J.: Power laws, pareto distributions and zipf's law. Contemporary Physics 46(5), 323-351 (2005)

24. Tenenbaum, J.B., de Silva, V., Langford, J.C.: A Global Geometric Framework for Nonlinear Dimensionality Reduction. Science 290(5500), 2319-2323 (2000)

25. Gabriel, G., Maher, M.: Coding and modeling communication in architectural collaborative design. Automation in Construction 11, 199-211 (2002) 